\title{
Reflets
}

Revue ontaroise d'intervention sociale et communautaire

\section{La dialectique de l'économie sociale : travail, employabilité, solidarité}

\section{Paul Leduc Browne}

Volume 5, numéro 1, printemps 1999

Pratiques et développement économique communautaire

URI : https://id.erudit.org/iderudit/026248ar

DOI : https://doi.org/10.7202/026248ar

Aller au sommaire du numéro

Éditeur(s)

Reflets : Revue ontaroise d'intervention sociale et communautaire

ISSN

1203-4576 (imprimé)

1712-8498 (numérique)

Découvrir la revue

Citer cet article

Leduc Browne, P. (1999). La dialectique de l'économie sociale : travail,

employabilité, solidarité. Reflets, 5(1), 28-53. https://doi.org/10.7202/026248ar

Tous droits réservés (C) Reflets : Revue ontaroise d'intervention sociale et communautaire, 1999
Ce document est protégé par la loi sur le droit d'auteur. L'utilisation des services d'Érudit (y compris la reproduction) est assujettie à sa politique d'utilisation que vous pouvez consulter en ligne.

https://apropos.erudit.org/fr/usagers/politique-dutilisation/ 


\title{
La dialectique de l'économie sociale: travail, employabilité, solidarité
}

\author{
Paul Leduc Browne
}

Centre canadien de politiques alternatives

\section{Une économie politique attendrie? Fonctions de l'économie sociale}

«La popularité

croissante de la notion

d'économie sociale

reflète à la fois son

ambiguïté et son

potentiel comme

stratégie de changement socio-économique.»
L'économie sociale, comme les thèmes apparentés du développement économique communautaire (DÉC), de l'économie solidaire, du développement local et du tiers-secteur, occupe depuis quelques années une place toujours grandissante dans la théorie, la stratégie et les politiques socioéconomiques, tant des instances gouvernementales fédérales, provinciales et municipales, que des interventions du milieu communautaire. La popularité croissante de la notion d'économie sociale reflète à la fois son ambiguïté et son potentiel comme stratégie de changement socio-économique.

Par économie sociale, on entend l'univers des pratiques et des formes de mobilisation qui ne font partie ni de l'entreprise capitaliste, ni des appareils de l'État au sens étroit du terme. Ce sont principalement les secteurs associatif, bénévole et coopératif (Quarter 1992), dont les organismes membres ont des objectifs et des structures de fonctionnement démocratiques, autonomes, sans but lucratif et au service de la collectivité (Comité d'orientation 1996). Formellement indépendants par rapport à l'État, leurs activités marchandes sont perçues comme des moyens de réaliser des objectifs de développement social qui dépassent la simple logique de marché. 
«Parler d'économie sociale plutôt que de tiers-secteur offre aussi l'avantage de ne pas créer l'illusion qu'il existe des "secteurs» nettement distincts.»
Cette définition résume des caractéristiques essentielles attribuées à l'économie sociale (Lévesque et Ninacs 1997) et la rapproche de certaines définitions du tiers-secteur (Salamon 1994; Browne 1999a). Il est important de noter que la notion d'économie sociale met l'accent sur la dimension économique, ce que le concept de tiers secteur ne fait pas. Salamon, par exemple, n'y inclut pas les coopératives, mais y situe les universités. La notion d'économie sociale met en relief aussi la primauté des objectifs sociaux et des structures démocratiques, alors que le concept de tiers secteur est souvent défini en fonction de critères juridiques (par exemple, le statut charitable ou sans but lucratif des organismes), sans tenir compte de la nature des rapports sociaux au sein des organismes, de leurs structures de prise de décision ou de leur enracinement dans les communautés locales. Parler d'économie sociale plutôt que de tiers-secteur offre aussi l'avantage de ne pas créer l'illusion qu'il existe des «secteurs» nettement distincts. Il s'agit plutôt de concevoir l'économie sociale comme un ensemble de réseaux regroupant des acteurs venant tant des coopératives et des organismes sans but lucratif que d'agences gouvernementales et d'entreprises privées qui en assurent le financement.

Pour les gouvernements animés d'un esprit néo-libéral, l'économie sociale représente d'une part un pont entre le chômage et l'emploi, une stratégie intéressante pour récupérer ceux et celles dont la réintégration au marché du travail est obstruée par un déficit d'«employabilité», c'est-à-dire par un manque de formation, des handicaps physiques ou mentaux, etc. Dans le contexte de l'économie de marché, le travail salarié dans le secteur privé à but lucratif, ou à la rigueur dans le secteur public, demeure le seul modèle jugé désirable. Toutes les autres formes de participation au travail doivent au plus y suppléer. D'autre part, l'économie sociale offre un moyen de réorganiser les services à la population. Dans une optique néo-libérale il s'agit

de rétrécir l'offre publique de services en transférant des responsabilités accrues au secteur privé (privatisation), au secteur associatif (communautarisation) et au secteur informel (familialisation), dans le but principal de réduire les cô̂ts sans se préoccuper de la qualité des 
services et des emplois concernés. Il s'agit d'une stratégie du déversoir. L'État recule et transmet des lots de responsabilités au marché, aux familles et à la communauté dans le seul but de réaliser des économies de coûts (Vaillancourt 1997: 248).

"Pour d'autres cependant, l'économie sociale s'inscrit dans une vision bien différente de la société. Sa mission est bien moins de consolider à la marge l'économie de marché en récupérant, par exemple, les chômeurs, que d'élargir l'éventail des modèles économiques afin de mettre le développement économique au service $d u$ développement social.»
Pour d'autres cependant, l'économie sociale s'inscrit dans une vision bien différente de la société. Sa mission est bien moins de consolider à la marge l'économie de marché en récupérant, par exemple, les chômeurs, que d'élargir l'éventail des modèles économiques afin de mettre le développement économique au service du développement social. L'économie sociale vise alors à développer et à consolider de façons plurielles les ressources d'une communauté, ainsi que de la rendre plus autarcique.

D'après la tradition tocquevillienne, les associations renforcent la participation démocratique - il faut à cet égard évoquer l'oeuvre récente de Robert Putnam, qui connaît un grand retentissement (Putnam 1993, 1994, 1995, 1996a, 1996b; Lemann 1996). Selon Putnam, la densité des réseaux communautaires et le foisonnement de la vie associative constituent la variable indépendante qui explique à la fois le dynamisme des économies modernes complexes et le succès des régimes politiques démocratiques. Là où cette variable est faible, la démocratie est chétive et l'économie stagnante. Certains vont jusqu'à faire de la «société civile» le véhicule par excellence de la démocratie, de la convivialité, de l'altruisme, de la coopération, de l'innovation. Sans nier le potentiel qu'elle recèle, il ne faut pas cependant confondre l'idéal et l'empirique. Theda Skocpol (1996) a démontré le rôle de l'État dans l'essor de la société civile elle-même. Il est évident que les secteurs associatif et coopératif entretiennent depuis le $19^{\text {e }}$ siècle une relation complexe avec l'État. De plus, la montée de l'État-providence n'a pas signifié l'éclipse de la philanthropie, du bénévolat ou du communautaire - bien au contraire. Ce tiers secteur a connu une croissance remarquable sous l'égide de l'État-providence. Il y a toujours eu, et il y a toujours, en Europe de l'Ouest et en Amérique du Nord, ce que les Britanniques appellent le «mixed economy of welfare» (RCPS 1998; Maurutto 1998; Browne 1996; Castel 1995; Finlayson 1994; Rekart 1993). 
Toutefois, le rôle de l'économie sociale a évolué en fonction de la trajectoire de l'État-providence lui-même (Browne 1996).

Quant au rôle de la vie civique et associative dans le fonctionnement démocratique, c'est une réalité qu'il faut nuancer. La vie associative peut tout autant représenter un instrument de mobilisation de forces hostiles à la démocratie. L'historien Rudi Koshar (1986) a par exemple démontré le rôle du monde associatif allemand dans la montée du nazisme. Au Canada, des associations telles que le Business Council on National Issues, l'Institut Fraser, l'Institut C.D. Howe, le National Citizens' Coalition, le Canadian Taxpayers' Federation et la Chambre de Commerce du Canada, sont le fer de lance de l'offensive néo-libérale (Clarke 1997; Kelsey 1995).

L'essentiel est sans doute que le thème de l'économie sociale suscite une interrogation de la nature, du sens et des finalités du travail. Qu'est-ce que la richesse? Comment la produire? Comment la répartir? Comment faire en sorte que chacun et chacune puisse participer de façon démocratique à la vie politique et économique? Ces questions sont d'autant moins abstraites qu'elles sont explicites dans la démarche des divers acteurs étatiques et communautaires qui prônent une stratégie de DÉC. L'intérêt que suscite celui-ci est justement lié intimement à l'ajustement structurel de l'économie nationale et au sort de l'État-providence.

«Le DÉC et

l'économie sociale apparaissent comme un vecteur essentiel pour répondre à la crise de l'emploi et à celle, corrélative, de l'État-providence.»
Le DÉC et l'économie sociale apparaissent comme un vecteur essentiel pour répondre à la crise de l'emploi et à celle, corrélative, de l'État-providence. Le concept de l'économie sociale a justement quitté les salles de séminaire pour faire irruption dans les manchettes à la faveur de la Marche des femmes contre la pauvreté Du pain et des roses en juin 1995 et du Sommet de l'emploi au Québec convoqué à l'automne 1996 par le premier ministre Bouchard. Dans un tel contexte, le regain d'intérêt pour l'économie sociale n'a rien de surprenant. Au début du siècle dernier, l'économiste français Sismondi affirmait déjà la nécessité de corriger l'orientation exclusive de l'économie politique vers la cupidité, au moyen d'une économie sociale axée sur le bien commun. En 1898, René Worms déclarait: «L'économie sociale est une économie politique attendrie» (Cité par Castel 1995 :245). 
Si l'on parle de nouveau d'économie sociale aujourd'hui, n'estce pas parce que l'économie de marché s'est tellement endurcie que sa déshumanisation est devenue flagrante? L'appauvrissement, l'inégalité croissante, l'idéologie néo-libérale, les politiques socioéconomiques rétrogrades rappellent le capitalisme sauvage du $19^{e}$ siècle. Comme jadis, l'économie sociale se veut l'incarnation du côté humain de la société.

\section{La crise de la société salariale et la notion néo-libérale de l'employabilité}

La fin de la Deuxième Guerre mondiale a inauguré une période d'une trentaine d'années de croissance économique tout à fait extraordinaire dans tous les pays industrialisés. Cet essor a jeté les fondements de l'État-providence, axé sur le couple plein emploi salarié des hommes/travail domestique des femmes et son corollaire, le couple assurance sociale/aide sociale. C'est le modèle de la «société salariale» (Laville 1994 : 48 et suiv.). Benoît Lévesque le décrit ainsi :

Il s'agissait d'un projet de société où le statut de citoyen de plein droit était réservé au titulaire d'un emploi salarié, une société où une part de plus en plus importante des droits était rattachée au statut de salarié à travers non seulement le salaire direct mais aussi le salaire indirect: avantages sociaux, vacances, retraite, etc. Une société où théoriquement tous ceux et toutes celles qui le désireraient pourraient exercer un travail rémunéré, c'est-à-dire un emploi. (1995:19)

Comme le fait remarquer Castel, ce modèle social désindividualise le travailleur en l'inscrivant «dans des régimes généraux, conventions collectives, régulations publiques du droit du travail et de la protection sociale». À la masse d'individus atomistiques interchangeables caractérisant un capitalisme "pur», le modèle de 
la société salariale substitue "un emboîtement hiérarchique de collectivités constituées sur la base de la division du travail et reconnues par le droit» (Castel 1995 : 466-467). Dans ce modèle, les gouvernements sont tenus de réaliser le plein emploi et de fournir à tous et à toutes une protection universelle contre les risques associés au chômage, à la maladie et à la vieillesse. Cette protection doit prendre la forme de l'accès universel à la sécurité du revenu (sous les formes de régimes de rentes et d'assurances sociales), à l'assurance-maladie et à l'éducation publique. Les citoyens jouissent ainsi, du moins en principe, de droits sociaux et économiques qui font en sorte qu'ils ne sont condamnés ni à la misère, ni à vivre aux dépens de leur famille ou de la bienfaisance privée. C'est dans la reconnaissance de ces droits que le Canada a signé le Pacte international sur les droits économiques, sociaux et culturels, qui complète la Déclaration universelle des droits humains de l'ONU.

"Pourl'essentiel, cependant, la société salariale est fondée, comme toute société capitaliste, sur le rapport de production entre le capital et le travail. C'est un système économique où la grande majorité de la population ne possède et ne contrôle pas les moyens de produire ce qu'il lui faut pour survivre et mener une vie civilisée.»
Pour l'essentiel, cependant, la société salariale est fondée, comme toute société capitaliste, sur le rapport de production entre le capital et le travail. C'est un système économique où la grande majorité de la population ne possède et ne contrôle pas les moyens de produire ce qu'il lui faut pour survivre et mener une vie civilisée. Pour ce faire, elle doit vendre sa force de travail à ceux qui détiennent ces moyens de production.

Or, vers le milieu des années 1970, la baisse généralisée des taux de profit des entreprises et le ralentissement de la croissance exacerbent les conflits entre le patronat et le mouvement ouvrier. On voit se dessiner du côté des entreprises un nouveau modèle de développement - le "post-fordisme» - caractérisé par de nouvelles orientations des entreprises vers les marchés et de nouvelles formes d'organisation et de gestion : remise en question de la production de masse de marchandises standardisées pour des marchés homogènes, production à plus petite échelle d'une variété de produits, sous-traitance, flexibilité de la main-d'oeuvre, tâches et qualifications multiples, travail en équipe, etc. (Harvey 1988; Lipietz 1989; Jenson et al. 1993). Tout cela s'accompagne de la rentrée massive des femmes sur le marché du travail, de la montée du chômage, d'une forte augmentation du pourcentage de travailleurs temporaires ou à temps partiel, ainsi que des 
"Rifkin propose la création d'un vaste tiers secteur en dehors du marché, qui accomplirait des tâches d'utilité sociale collective. Cette économie sociale mettrait au travail les personnes sousemployées ou en chômage, qui ne toucheraient pas de salaire, mais recevraient de l'État un revenu annuel garanti.» travailleurs autonomes. Le plein emploi prend fin et la société salariale est en crise.

Par extrapolation de tendances semblables aux États-Unis, Jeremy Rikin (1994) est arrivé à l'hypothèse de la fin du travail (pour une mise au point sur la question, voir Jackson, 1997). Selon Rifkin, l'économie de marché ne pourra plus créer à l'avenir suffisamment d'emplois réguliers à temps plein pour toute la population active, en raison des changements technologiques. Pourtant, de nombreux besoins sociaux resteront inassouvis. Rifkin propose la création d'un vaste tiers secteur en dehors du marché, qui accomplirait des tâches d'utilité sociale collective. Cette économie sociale mettrait au travail les personnes sous-employées ou en chômage, qui ne toucheraient pas de salaire, mais recevraient de l'État un revenu annuel garanti.

Quoi qu'on puisse penser de la futurologie d'un Rifkin - et il est certain qu'il existe une quantité énorme de besoins sociaux inassouvis, ainsi qu'un réservoir immense de travail potentiel dans la société, et que l'idée d'un revenu annuel garanti mérite d'être débattue (le livre de Rifkin est d'ailleurs d'un grand intérêt) -, il reste que le ratio emploi/population a eu tendance à croître au cours du dernier demi-siècle, en même temps que le taux de chômage ${ }^{1}$. En effet, il est frappant de constater que le pourcentage des Canadiens et des Canadiennes âgés de plus de quinze ans occupant un emploi salarié a en fait augmenté depuis un demisiècle. Du milieu des années cinquante au milieu des années soixante, environ $50 \%$ des Canadiens et des Canadiennes âgés de quinze ans et plus étaient salariés. Cette proportion a par la suite augmenté de façon graduelle pour plafonner à 62,4 \% en 1989. En 1995, après plusieurs années de récession, le ratio emploi/ population se situait à $58,6 \%$. Il faut cependant noter que la situation des femmes differe de fond en comble de celle des hommes. En 1946, 87 \% des hommes étaient salariés; ce pourcentage n'était que de 77,8 \% en 1976 et de 68,1\% en 1995. Cependant le pourcentage des femmes salariées a triplé entre 1946 et 1995, passant de 17,9\% en 1946 à 32,9\% en 1970 et à $52,1 \%$ en 1995. On a ainsi assisté à une forte hausse du taux d'activité (c'est-à-dire de l'ensemble des salariée-s et des personnes 
"On n’a pas assisté à la transition d'un plein emploi absolu à une disette absolue d'emplois, mais plutôt d'un plein emploi relatif à un chômage relatif grandissant.» à la recherche d'un emploi). Dans les années cinquante, le taux d'activité se situait en moyenne dans les $53 \%$; il a plafonné en 1989 à 67,5\% et était de $65 \%$ vers la fin de 1997 (Akyeampong 1996; Statistique Canada 1998: 16).

Si le plein emploi des hommes est chose du passé, le taux d'activité des femmes a fortement augmenté, tout en n'égalant pas encore celui des hommes. Il est donc prématuré de conclure à la fin du salariat. Toutefois, on peut évoquer une crise de la société salariale (Favreau et Lévesque 1996; Lévesque 1995; Castel 1995). On n'a pas assisté à la transition d'un plein emploi absolu à une disette absolue d'emplois, mais plutôt d'un plein emploi relatif à un chômage relatif grandissant.

Selon des agences internationales telles que l'OCDE et le FMI, à défaut d'augmentations importantes de la productivité, les niveaux de salaire élevés et les prestations généreuses de sécurité du revenu de nombreux pays industrialisés ne sont plus réalistes dans un marché mondial où la concurrence devient de plus en plus féroce. La syndicalisation, les dépenses sociales, la réglementation des salaires par l'État, des niveaux insuffisants de formation et d'adaptation au changement des travailleurs - tous ces facteurs constitueraient des obstacles à la hausse de la productivité et contribueraient au chômage (OCDE 1995; Banque mondiale 1995).

Faisant écho à ces idées, le ministère fédéral des Finances attribua la montée «du taux de chômage fondamental» depuis les trente dernières années à quatre facteurs: «une mésadaptation croissante des compétences des travailleurs aux exigences du marché du travail, les effets désincitatifs exercés par les divers programmes de soutien du revenu ainsi que certains règlements régissant le marché du travail, l'alourdissement des charges sociales et la gravité exceptionnelle des deux dernières récessions» (Martin 1994 : 23). Le rapport entre la vision de l'OCDE et celle de Paul Martin est commenté par Jackson 1995; Régimbald 1995 et Stanford 1995.

Selon cette optique, le chômage présente deux caractéristiques clés. Tout d'abord, les individus demeurent en chômage de plus en plus longtemps. Cela n'affecte pas seulement leur bien-être 
"Quand le chômage se prolonge, on assiste souvent à une diminution sensible des qualifications professionnelles et à une perte de confiance, ce qui contribue à réduire les chances de se retrouver un emploi lorsque la reprise se produit» économique, mais également leurs chances de s'en tirer à l'avenir : "Quand le chômage se prolonge, on assiste souvent à une diminution sensible des qualifications professionnelles et à une perte de confiance, ce qui contribue à réduire les chances de se retrouver un emploi lorsque la reprise se produit" (Martin 1994 : 24-25). Par conséquent, «les chômeurs de longue durée sont de plus en plus des personnes qui ne possèdent pas les aptitudes requises et n'ont pas une souplesse suffisante pour acquérir de nouvelles compétences - ou n'ont pas l'occasion de le faire» (Martin 1994 :28). En raison des difficultés qu'ils éprouvent dans leur recherche d'un emploi, ils «risquent de devenir aliénés et de ne plus croire à la justice ni à aux valeurs fondamentales de la société» (Martin 1994:26). La réponse n'est donc pas seulement de réduire les prestations de sécurité du revenu, les niveaux des salaires et les protections syndicales, mais aussi de mettre en oeuvre des «mesures actives» afin d'accélérer et de faciliter la réinsertion des chômeurs sur le marché du travail. Bien entendu, une telle stratégie se voit opposer l'inutilité de préparer les individus à réintégrer le marché du travail s'il y a une pénurie d'emplois. À cela, le ministère des Finances donne la réplique suivante:

S'il est vrai que la demande globale a été insuffisante dans l'économie canadienne depuis le début de la récession en 1990, il importe de reconnaître que, même dans un marché du travail qui traverse le creux $d u$ cycle économique, on observe toujours un "roulement" extrêmement élevé. En moyenne, plus de 20 pour cent des travailleurs canadiens (environ 2,5 millions) quittent leur emploi chaque année en démissionnant ou en étant mis à pied de façon permanente, tandis qu'une proportion légèrement supérieure obtient habituellement un emploi. Cela signifie que le roulement de la population active nationale se fait de façon continue, bien que pas dans des localités particulières. Ainsi, même s'il y aura inévitablement des personnes qui, malgré la meilleure intention, ne se trouveront pas un travail, ceux ou celles qui sont suffisamment souples ont une meilleure chance de se décrocher un emploi 
grâce au roulement continu observé sur le marché $d u$ travail, même lorsque l'activité économique est faible (Martin 1994 : 26).

"La crise de l'emploi et la crise fiscale de l'État servent de prétexte au démantèlement des fondements $d u$ providentialisme, dont les assurances sociales et les pensions universelles...»
Étant donné le roulement de la population active, des niveaux de chômage élevés en soi ne condamnent personne à une vie sur le bien-être social. Au contraire, la réinsertion dans l'emploi est probable, à condition d'être employable, c'est-à-dire doté d'habiletés, confiant, adaptable, prêt à toute éventualité. C'est donc aux travailleurs de rehausser leur degré d'employabilité et aux gouvernements de les encourager à le faire.

À partir de 1994, le gouvernement fédéral libéral va mettre en place un nouveau modèle de gestion étatique des conflits et des problèmes sociaux, dont la fonction est de créer les conditions de réalisation de ce nouveau modèle économique. La plupart des provinces vont emboiter le pas. On assistera dès lors à une véritable offensive contre toutes les conditions sociales, culturelles, juridiques et politiques qui avaient caractérisé le compromis social-démocrate des décennies précédentes. La crise de l'emploi et la crise fiscale de l'État servent de prétexte au démantèlement des fondements du providentialisme, dont les assurances sociales et les pensions universelles (régimes de retraite et allocations familiales). Dans l'esprit du néo-libéralisme, les gouvernements retournent à la politique sociale du siècle dernier. Ils proclament la nécessité de laisser libre cours aux forces du marché, auxquelles ils promettent de livrer les chômeurs et les personnes dans le besoin pour leur plus grand bien (Campbell et Barlow 1995; McQuaig 1993; Ralph et al.1997). Ils entament le grand virage néo-libéral et monétariste (Mishra 1990; Block et al. 1987; Hall et Jacques 1983). Une politique monétaire draconienne, accompagnée de réformes fiscales régressives, entraîne la croissance vertigineuse de la dette de l'État, en même temps qu'elle mine la croissance économique, exacerbant le chômage et affaiblissant les salariés face au patronat (AFB 1998; Stanford 1997a, 1997b).

Dans ce contexte, l'économie sociale séduit en tant que véhicule potentiel de réinsertion dans l'emploi par le biais du développement des ressources humaines. Comme la notion de 
"Les gouvernements ont beaucoup misé sur les organismes appartenant à l'économie sociale comme instruments de telles politiques d'employabilité." "capital humain», celle du développement des ressources humaines envisage l'activité humaine du point de vue de la science économique comme un facteur de production dont les êtres humains sont essentiellement les propriétaires. En tant qu'acteurs économiques, les individus doivent obéir à l'impératif de maximiser la valeur de leur propriété et d'en tirer le plus grand rendement possible. La notion d' «employabilité» joue un rôle clé dans cette perspective, car elle combine les divers aspects du développement des «ressources humaines» dans le but d'en maximiser la valeur marchande. Parmi ces aspects on compte les habiletés et les qualifications; la participation à des réseaux sociaux ayant une pertinence dans la recherche d'un emploi; la confiance en soi et l'estime de soi; l'ambition, l'initiative, la volonté de réussir; la vision de l'avenir. Les mesures d'employabilité comprennent par conséquent diverses formes d'éducation et de formation, de même que d'autres pratiques qui relèvent du service social et de la psychologie.

Les gouvernements ont beaucoup misé sur les organismes appartenant à l'économie sociale comme instruments de telles politiques d'employabilité. Ces organismes offrent souvent une formation à leurs employés et à leurs bénévoles. Ils sont plus susceptibles d'embaucher des personnes qui ne sauraient accéder au secteur privé et de leur inculquer des habiletés techniques et autres, une éthique et des habitudes de travail, une bonne estime de soi, soit l'expérience professionnelle que recherche tout employeur. De tels placements peuvent être conçus comme menant directement à un emploi régulier (comme dans le cas de l'ancien programme BoulotOntario), ou temporaires (comme dans le cas du programme «Ontario au travail» ou des CIT au Québec). Ils peuvent payer un supplément aux salaires offerts par les employeurs, payer le salaire minimum, n'offrir que l'équivalent de la prestation d'aide sociale ou, comme dans le cas d' "Ontario au travail», comprendre une dimension de «bénévolat forcé», c'està-dire de travail obligatoire (workfare) (Browne 1999b;Vosko 1999).

Dans la logique de l'employabilité, l'individu est sur la sellette quand il s'agit de se trouver un emploi. La métaphore d'un jeu de chaises musicales où chacun et chacune occupe un emploi une 
«La pénurie d'emplois engendre la concurrence, donc l'efficacité, contraignant les salariés à redoubler d'ardeur en retour d'une rémunération plus faible. Selon cette logique, chacun et chacune se doit d'améliorer la qualité de sa marchandise et de chercher plus agressivement à la vendre.» partie du temps, sinon constamment, se joint à la maxime libérale politique de l'égalité des chances, à la maxime libérale économique des bienfaits de la concurrence et à la maxime libérale éthique de la moralité intrinsèque du travail. Le roulement de la population active crée des chances d'emploi. La pénurie d'emplois engendre la concurrence, donc l'efficacité, contraignant les salariés à redoubler d'ardeur en retour d'une rémunération plus faible. Selon cette logique, chacun et chacune se doit d'améliorer la qualité de sa marchandise et de chercher plus agressivement à la vendre. Puisque l'égalité des chances règne, et que l'égalité des chances est synonyme de justice, cette situation n'a donc rien d'injuste. L'impossibilité du plein emploi excuse le chômage de courte durée. Mais dans cette optique, selon laquelle l'oisiveté est immorale, le chômage de longue durée sent la turpitude.

Le chômage résulterait alors de la paresse ou d'une déficience d'employabilité que l'individu aurait le devoir de corriger. Le corollaire en est que les chômeurs sont coupables d'une certaine faute morale - ils n'ont pas fait suffisamment d'efforts, ou n'ont pas fait preuve de l'intelligence nécessaire pour s'en sortir - et que l'État peut donc en toute légitimité les soumettre à un régime punitif ou coercitif. Il peut ainsi les priver de certains droits (les prestataires de l'aide sociale participant au programme «Ontario au travail» n'ont par exemple pas le droit de se syndiquer); il peut pratiquer une discrimination à l'égard de certains individus (par exemple, les prestataires «fréquents» de l'assurance-emploi dont les prestations sont réduites); il peut les assujettir à des mesures quasi-policières (les contrôler en prélevant leurs empreintes digitales).

Plutôt que d'analyser le chômage comme résultat de conditions et de politiques macroéconomiques, cette optique néolibérale met ainsi tout l'accent sur le comportement des individus. Il ne peut s'agir de structurer le marché selon les besoins des individus; au contraire, ceux-ci doivent se conformer à la logique du marché (Larochelle et Tremblay 1994; Provost 1989). Toute analyse critique des institutions et des structures économiques est mise de côté en faveur d'une évaluation psychologique des individus. Les problèmes n'apparaissent plus comme objectifs dans 
"Dans une optique néo-libérale, voilà après tout deux formes apparentées de déviance, qu'on peut traiter d'objets semblables de régulation morale et de manipulation scientifique." cette optique, mais comme subjectifs, comme carences d'estime de soi, de motivation, d'adaptabilité, de compétitivité, d'initiative, de pensée positive. Les individus taxés de «déficience d'employabilité» font alors l'objet non seulement de sanctions financières et d'efforts de régulation morale, mais aussi de mesures sociales et psychologiques de modification du comportement aux mains d'agences gouvernementales et non-gouvernementales chargées d'exécuter les politiques publiques. Que l'on ait utilisé l'expression «travail communautaire» (community work) pour décrire à la fois les programmes mis en place pour les criminels et ceux destinés aux prestataires de l'aide sociale (notamment dans le cadre d' «Ontario au travail») n'est pas un accident. Dans une optique néo-libérale, voilà après tout deux formes apparentées de déviance, qu'on peut traiter d'objets semblables de régulation morale et de manipulation scientifique.

Le néo-libéralisme propose une logique de démantèlement des services sociaux: sortant de l'orbite étatique, ils sont réintégrés dans le secteur privé s'ils trouvent des entrepreneurs voulant les organiser ou ils prennent place dans les activités sociales informelles si tel n'est pas le cas. La stratégie marchande assimile les demandes d'autodétermination émanant des professionnels et des usagers à une revendication de libre entrepreneuriat. Quant à la stratégie en faveur des activités sociales informelles, elle met en avant le bénévolat (...). Toutefois, ce volontarisme élude les questions des ressorts de la coopération et du don, il évacue le lien entre le niveau d'emploi et de revenus de transfert et la propension à s'engager dans des actions volontaires. Il cherche à restaurer un état social antérieur où de multiples services sociaux étaient assurés par la famille et le voisinage sans s'attarder sur les modifications culturelles enregistrées depuis lors. Son postulat implicite est la stabilité des rôles familiaux et sexuels, et c'est fort logiquement que les politiques conservatrices incitent les femmes à quitter le marché du travail. Si le rôle de l'Etat s'estompe, la baisse de la protection sociale et 
des rémunérations peut certes favoriser l'offre de services mais avec pour horizon la "société de serviteurs" qu'évoque Gorz, les femmes étant les premières à voir leur situation se dégrader, obligées de choisir entre le statut dépendant du travail domestique et un emploi faiblement rémunéré, précaire et à temps partiel (Laville 1993: 186).

«...la promotion $d u$ communautaire, $d u$ bénévolat, de l'économie sociale, peut très bien servir le projet néo-libéral de restructuration de l'État..."
Dans ce contexte, il est évident que la promotion du communautaire, du bénévolat, de l'économie sociale, peut très bien servir le projet néo-libéral de restructuration de l'État, de démantèlement de l'État-providence, d'assaut contre les syndicats $\mathrm{du}$ secteur public - et donc d'offensive contre les salaires, les protections sociales et le niveau de vie des travailleurs. À noter par exemple la promotion du bénévolat et de la philanthropie par les Reagan, George Bush père, Mike Harris et Paul Martin. Il n'est donc guère étonnant que l'économie sociale fasse l'objet de soupçons, voire d'hostilité, notamment dans le milieu syndical, tant au Québec que dans le reste du Canada.

\section{Vers une économie solidaire}

Cela étant dit, l'évocation du scénario néo-libéral n'épuise pas la question. La critique de la société salariale n'est pas venue que de la droite (Laville 1994: 55). Le dernier quart de siècle a aussi connu l'émergence d'une foule de mouvements sociaux luttant d'une part pour de nouvelles formes de redistribution, d'autre part pour la reconnaissance de leurs identités naguère inconnues ou méconnues. Parallèlement à la crise des modèles universalisants dans les domaines économiques et sociaux (plein emploi, production et consommation de masse de produits et de services standardisés, programmes sociaux universels), on a assisté à une crise de l'universalisme aux niveaux politique et culturel (démocratie réduite au simple suffrage universel, systèmes juridiques universels et abstraits ne tenant pas compte des vraies 
"Toute une gamme

d'auteurs et de militants critiquent l'État-providence keynésien et proposent des modèles de développement communautaire et démocratique, afin de contrer le néolibéralisme et d'avancer vers un État et une société plus démocratiques, plus participatives, plus inclusives et plus justes.» différences au sein de la population, discours philosophiques et artistiques universels et homogénéisants). C'est l'émergence de la "condition postmoderne». L'État-nation doit répondre au défi de la mondialisation, de la montée des multinationales - mais aussi à celui de nouveaux mouvements de gauche privilégiant le territoire local ou régional, ou le regroupement identitaire, comme lieu d'action politique et de développement socio-économique.Toute une gamme d'auteurs et de militants critiquent l'État-providence keynésien et proposent des modèles de développement communautaire et démocratique, afin de contrer le néo-libéralisme et d'avancer vers un État et une société plus démocratiques, plus participatives, plus inclusives et plus justes.

Au niveau de la production des biens et des services, la société salariale a pendant quelques années réussi à répartir l'emploi de façon à redistribuer la richesse suffisamment pour garantir un revenu grandissant à la plupart des ménages. Cependant, il n'a pas pour autant émancipé les travailleurs, qui sont restés asservis à un système industriel hiérarchique, bureaucratique et autoritaire, où le travail représentait trop souvent la perte de soi (Linhart 1978; Braverman 1994). Dans ce système, comme le fait remarquer Benoît Lévesque, les niveaux grandissants de consommation sont censés compenser le caractère abrutissant du travail : «'exclusion dans l'organisation du travail donne droit à une participation dans le secteur de la consommation.» (Lévesque 1995 : 21) Cependant la consommation est elle-même aliénante. Elle atomise les individus, les séparant les uns des autres; elle fait de plus en plus passer leur interaction par l'entremise de relations commerciales. Et si les citoyens ont droit à l'accès universel aux services publics, ceux-ci sont eux-mêmes produits et distribués selon un système hiérarchique et bureaucratique, qui interdit une réelle participation tant aux usagers qu'aux producteurs de ces services eux-mêmes.

La consommation de masse (marchande) de même que celle (non-marchande) de services collectifs bureaucratisés produisaient la dépendance et la passivité et étaient déficientes sur le plan des relations sociales, de la socialisation et de la participation à la société. Autrement dit, la compensation par la consommation pour 
l'exclusion dans le travail s'est avérée elle-même passablement limitée, "excluante», voire productrice de nouvelles exclusions sociales. (...) De ce point de vue, la nécessité de repenser l'État-providence, de repenser les rapports entre l'économique et le social, s'imposerait même si la dette de l'État disparaissait (Lévesque 1995: 21).

"La société salariale, bien que préférable sous bien des angles au modèle néo-libéral, n'en reste donc pas moins un régime caractérisé par la hiérarchie et l'oppression des travailleurs, des femmes, des minorités ethniques, raciales et nationales...»
La société salariale, bien que préférable sous bien des angles au modèle néo-libéral, n'en reste donc pas moins un régime caractérisé par la hiérarchie et l'oppression des travailleurs, des femmes, des minorités ethniques, raciales et nationales (Ursel 1992; Amin 1976; Gorz 1978; Marcuse 1964; Mészáros 1970; Baran et Sweezy 1966). La société salariale constitue dans une certaine mesure l'utopie sociale-démocrate. C'est une vision qui ne remet pas en question le rapport capital-travail, mais cherche à créer un équilibre différent entre les deux. L'objectif social-démocrate est d'ailleurs d'élargir et de généraliser davantage le salariat, en créant beaucoup plus de salariés de l'État et en transformant de plus en plus le travail domestique et les autres formes de travail non rémunéré en travail salarié.

Reprenant les analyses de Polanyi (1957), Laville (1994;1993) met en perspective l'économie marchande et la société salariale en rappelant quatre grands principes de l'organisation économique: le marché, la redistribution, la réciprocité et l'administration domestique. Le marché représente un système où la production et la distribution de la terre, de la force de travail et des produits s'opère par l'entremise de l'échange des biens, lui-même médiatisé par un équivalent général (l'argent). La redistribution consiste en revanche à centraliser les biens en vue de leur redistribution par une autorité hiérarchique (l'État). L'administration domestique est la production et la gestion des ressources pour son propre usage ou celui de son groupe (la famille), alors que la réciprocité est fondée sur le don. Ces principes représentent évidemment des idéaux-types, qui ne sont ni présents empiriquement dans toute leur pureté, ni seuls présents dans une formation sociale donnée. Ils expriment cependant les idées-force de différents projets de société. C'est ainsi que le marché représente en quelque sorte la métaphore dominante du néo-libéralisme, la redistribution 
"Ils puisent dans leurs propres ressources de savoir et de sociabilité. Misant sur le bénévolat, l'entraide, la coopération, plutôt que sur la poursuite du profit, ils créent entre eux des réseaux de réciprocité." le principe essentiel de la social-démocratie et la réciprocité, le concept clé de l'économie solidaire.

Comme en Amérique du Nord, il existe des tendances vers une nouvelle «économie solidaire» dans plusieurs pays européens (Laville 1997, 1994, 1993). Face au chômage, à l'exclusion et à des secteurs publics incapables d'y répondre adéquatement, les travailleurs et les consommateurs des services de proximité (services aux personnes) forment de nouveaux réseaux pour subvenir à leurs besoins: soins à domicile, garde des enfants, soins de santé, transports en commun, culture, récréation, travail environnemental, coopératives de travailleurs et de consommateurs, systèmes locaux d'échange (local exchange trading systems). Ils puisent dans leurs propres ressources de savoir et de sociabilité. Misant sur le bénévolat, l'entraide, la coopération, plutôt que sur la poursuite du profit, ils créent entre eux des réseaux de réciprocité. Ce faisant, ils définissent et redéfinissent leurs besoins — le besoin de ce qui leur manque et le besoin de nouveaux modes d'y subvenir. Selon Jean-Louis Laville, ils en arrivent ainsi à construire de façon conjointe l'offre et la demande de services sociaux, plutôt que de se les laisser imposer. Ils créent aussi de nouveaux espaces publics de proximité, des espaces de discussion et de réflexion publique sur le développement social, où leurs valeurs, symboles et pratiques quotidiens jouent un rôle clef. Il s'agit de sortir les citoyens de leur situation passive de simples consommateurs de services publics conçus et gérés par des instances invisibles ou opaques. Il faut démocratiser les services pour libérer les producteurs et les consommateurs de l'emprise de structures de gestion régressives et leur permettre de définir conjointement l'offre et la demande des services. Enfin, on doit éviter que les familles, et surtout les femmes, soient accablées du fardeau des services que l'État ne peut plus et ne veut plus assumer. Ces prises de position débouchent sur la revendication de faire renaître la citoyenneté active, de reconstruire une société dense de ses réseaux de participation et d'appartenance, mais débarrassée des hiérarchies et tutelles patriarcales ou bureaucratiques.

Tout ceci s'apparente à la tradition autogestionnaire d'une démocratie construite à partir de la base, et Laville évoque d'ailleurs 
la riche tradition de la solidarité et de l'association ouvrière au XIX $^{\mathrm{e}}$ siècle (1994:23 et suiv.). Des penseurs et militants en Europe et en Amérique du Nord unissent la défense d'une économie solidaire à l'exigence d'une réduction et d'une redistribution généralisées du temps de travail salarié (32 HOURS 1998; Gorz 1997, 1988, 1978; Caillé 1995). Au départ, cette revendication se situe dans la logique du salariat. Les deux grandes revendications historiques des salariés sont l'augmentation des salaires et la réduction du temps de travail. Cependant, la lutte pour celle-ci peut se radicaliser, au point de remettre en question le pouvoir même du capital (Mészáros 1998).

Il s'agit aussi de reconnaître l'énorme apport économique du travail non rémunéré, et notamment du travail domestique. Les enquêtes sur l'emploi du temps ont démontré que les Canadiens et les Canadiennes consacrent une grande, parfois la majeure partie de leur temps au travail non rémunéré, soit le travail domestique d'éducation des enfants et d'activités ménagères, soit les activités bénévoles en dehors de la maison (Hall et al. 1998; Frederick 1993; Harvey et al. 1991; Duschene 1989). Il existe peu de statistiques à ce chapitre. En 1995, Statistique Canada a rapporté que le travail non rémunéré, si on lui attribuait une valeur marchande, vaudrait entre 234 et 374 milliards de dollars. Le travail non rémunéré au Canada représentait l'équivalent de 13 millions d'emplois, c'est-à-dire environ l'équivalent de la population «active» (donc salariée). Les femmes accomplissaient au moins deux-tiers du travail non rémunéré (Globe and.Mail 1995; cf. aussi Waring 1996, 1990; Chandler 1994; Frederick et al. 1992).

"Le modèle de l'économie solidaire ouvre la voie à une forme de reconnaissance et de protection de ceux et surtout de celles qui accomplissent les

travaux non

rémunérés»
Pour la plupart des Canadiens et des Canadiennes, le salariat reste la source principale des moyens de consommation nécessaires à la survie. Le salariat donne aussi accès aux meilleurs programmes sociaux, ceux basés sur les principes de l'assurance sociale (Assuranceemploi, Régime de pensions du Canada/Régime des rentes du Québec), alors que le travail non-rémunéré n'ouvre l'accès qu'aux programmes résiduels et paternalistes, tels que l'aide sociale.

Le modèle de l'économie solidaire ouvre la voie à une forme de reconnaissance et de protection de ceux et surtout de celles qui accomplissent les travaux non rémunérés (voilà aussi pourquoi 
"L'enjeu est plutôt de créer une nouvelle structure où la redistribution étatique et le salariat fonctionnent en synergie avec la réciprocité et le bénévolat, où les éléments du marché, de la redistribution et de la réciprocité se recombinent sous des formes nouvelles.» l'idée de l'économie solidaire occupait une place importante parmi les revendications de la Marche des femmes contre la pauvreté en 1995). Contre le néo-libéralisme qui voudrait refouler les femmes dans la domesticité pour compenser la perte des services publiques, il s'agit plutôt de les protéger du danger de devenir les principales ou seules productrices de services que l'État ne désire plus offrir - soins aux malades qui ne trouvent plus de lit à l'hôpital, bénévolat dans les écoles ou les hôpitaux en raison de la pénurie de personnel. Cependant, l'objectif n'est pas, selon Laville, de transformer les bénévoles en salariés et de remplacer la réciprocité par la redistribution étatique (l'option sociale-démocrate). L'enjeu est plutôt de créer une nouvelle structure où la redistribution étatique et le salariat fonctionnent en synergie avec la réciprocité et le bénévolat, où les éléments du marché, de la redistribution et de la réciprocité se recombinent sous des formes nouvelles.

C'est ainsi que Laville ne récuse pas l'État: «Ce n'est pas l'État qui est responsable de la dégradation sociale; au contraire sa présence a empêché qu'elle ne soit bien pire» (Roustang et al.1996: 80-81). L'État doit jouer un rôle très important. Cependant, ce rôle doit changer en fonction des nouvelles réalités économiques et sociales. La redistribution à elle seule ne suffit pas; il faut construire de nouvelles relations de réciprocité. Laville insiste sur la nécessaire «hybridation» des économies marchande, non-marchande et nonmonétaire, c'est-à-dire du marché, de l'État, du bénévolat, de l'entraide et de l'économie familiale. Il s'agit de rompre l'autarcie de l'économie de marché, la repolitiser, la réencastrer dans une société qui, elle, n'est pas réduisible au marché.

\section{Conclusion}

Tout en saluant cette perspective, je voudrais conclure par une mise en garde. L'économie solidaire est une possibilité, mais elle n'est pas encore une réalité bien ancrée. Devant sa réalisation se dresse l'obstacle d'un État néo-libéral au service de milieux financiers et économiques dont les intérêts sont loin de coïncider 
«Tant que tout le pouvoir économique et politique sera dans les mains d'une poignée de compagnies, l'économie sociale pourra-t-elle devenir davantage qu' «une forme d'autogestion de la pauvreté», qu'«un exercice d'autoexploitation", pour reprendre les mots de Gregory Baum?» avec ceux de la majorité. Cinquante et une des cent plus grandes entités économiques du monde sont des entreprises. Walmart, qui vient au douzième rang parmi les plus grandes compagnies, est plus grande que 161 pays, dont Israël, la Grèce et la Pologne. Les ventes combinées des 200 plus grandes firmes multinationales constituent plus du quart de l'activité économique totale du globe, soit 28,3 p. cent du PIB mondial. Il existe 191 États dans le monde. Si l'on retranche les neuf plus grands (États-Unis, Japon,Allemagne, France, Italie, Royaume-Uni, Brésil, Canada et Chine), les PIB combinés des 182 pays restants sont inférieurs aux ventes combinées des 200 plus grandes firmes multinationales (Anderson et Cavanaugh 1997: 12).

Tant que tout le pouvoir économique et politique sera dans les mains d'une poignée de compagnies, l'économie sociale pourra-t-elle devenir davantage qu' «une forme d'autogestion de la pauvreté», qu' «un exercice d'auto-exploitation», pour reprendre les mots de Gregory Baum (1989)? Pour le moment, l'économie sociale continue à vivre la contradiction évoquée par Louis Favreau, Benoît Lévesque et d'autres: si les groupes populaires veulent conquérir «de nouveaux espaces de liberté contre un pouvoir technocratique imposant ses choix de société» (Bélanger et Lévesque 1987 :260), il est tout aussi vrai que l'État «se décharge ... de ses responsabilités sur les groupes populaires, d'entraide» (Favreau 1989: 5); il les réduit au statut de sous-traitants dans le nouvel ordre néo-libéral. Telle est la dialectique de l'économie sociale: à la fois mouvement cherchant à bâtir des communautés plus humaines, plus conviviales, plus démocratiques, plus inclusives - et rouage dans la constitution néo-libérale de nouveaux modes de désamorcement et de gestion de l'exclusion.

Laville remet à juste titre en question l'antinomie du salariat et du bénévolat. Il est évident que celui-ci n'existe qu'en fonction de celui-là. Pour les syndicats, pour la Gauche, le recours au bénévolat dans les services publics et communautaires semble ouvrir la porte toute grande à la dégradation du salariat. Si le néo-libéralisme veut tout réduire au marché et exploiter les chômeurs soumis aux corvées du workfare, tout en chargeant le secteur informel des tâches qui ne sont pas rentables, la social- 
«Il faudra bien plus qu'un «tiers secteur» pour humaniser une économie mondiale lancée éperdument vers la tragédie écologique et la misère humaine.» démocratie veut réglementer le marché et maintenir le cloisonnement rigide de ce qui relève du travail rémunéré et du travail non-rémunéré, afin d'éviter ce genre d'exploitation. À la limite, le travail non-rémunéré serait limité à l'administration domestique et la réciprocité serait résiduelle (Laville 1993: 190). Parce qu'il remet au moins partiellement en question le salariat, Laville formule une vision potentiellement plus radicale que la social-démocratie. Toutefois, la question reste de savoir s'il est possible de promouvoir et de réaliser la solidarité au sein de la société néo-libérale.

La volonté d'humaniser la société de marché, en lui greffant de nouvelles institutions fondées sur la réciprocité, fait écho au rêve social-démocrate d'humaniser l'économie au moyen de la redistribution étatique. Reprenant la formule de Rosanvallon (1981), on pourrait baptiser de «postsocial-démocrate» ce mouvement vers une économie solidaire. Comme la social-démocratie, cependant, la post-social-démocratie vivra une situation profondément contradictoire: elle lutte pour l'équité,l'égalité et la réciprocité, sans toutefois remettre assez profondément en question les rapports de propriété dans un monde où la part de lion de la richesse - et du pouvoir - est aux mains de quelquesuns.

L'économie ne deviendra jamais vraiment solidaire tant qu'elle ne restera que locale, tant que des espaces publics plus vastes ne viendront pas s'ajouter aux espaces publics de proximité, tant que les principes démocratiques et coopératifs ne dépasseront pas le tiers secteur pour envahir les secteurs publics et privés. Il faudra bien plus qu'un «tiers secteur» pour humaniser une économie mondiale lancée éperdument vers la tragédie écologique et la misère humaine. Il faudra que l'économie et l'État dans leur ensemble se transforment en une "économie sociale des producteurs libres et associés» (Marx, cité par Mészáros 1995). 


\section{Bibliographie}

32 HOURS (1998). BetterTIMES :The Quarterly Newsletter of 32 HOURS :Action for Full Employment and the Shorter Work Time Network of Canada, Toronto.

AFB (1998). Alternative Federal Budget Papers 1998, Ottawa, Centre canadien de politiques alternatives/ CHO!CES : A Coalition for Social Justice.

AKYEAMPONG, Ernest B. (1996). "Another Measure of Employment», Perspectives on Labour and Income, Ottawa, Statistique Canada, hiver.

AMIN, Samir (1976). L'impérialisme et le développement inégal, Paris, Éditions de minuit.

ANDERSON, Sarah et John CAVANAGH (1997). «The Top 200 : The Rise of Global Corporate Power», CCPA Monitor, février, 12.

BANQUE MONDIALE (1995). Workers in an Integrating World, New York, International Bank for Reconstruction and Development.

BARAN, Paul et Paul SWEEZY (1966). Monopoly Capital, New York, Monthly Review Press.

BAUM, Gregory (1989). «L'Économie alternative», Relations, no. 548, mars, 41.

BÉLANGER, Paul R. et Benoît LÉVESQUE (1987). «Le mouvement social au Québe : continuité et rupture (1960-1985)", Animation et culture en mouvement. La fin d'une époque?, s. la dir. de Paul R. Bélanger, Benoît Lévesque, Réjean Mathieu and Franklin Midy, Sillery, Presses de l'Université du Québec.

BELLEMARE, Diane et Lise POULIN-SIMON (1994). What Is the Real Cost of Unemployment in Canada?, Ottawa, Centre canadien de politiques alternatives, mai.

BLOCK, Fred, Richard CLOWARD, Barbara EHRENREICH et Frances FOX PIVEN (1987). The Mean Season. The Attack on the Welfare State, New York, Pantheon Books.

BRAVERMAN, Harry (1974). Labor and Monopoly Capital, New York, Monthly Review Press.

BROWNE, Paul Leduc (1999a). «Le «tiers-secteur» au Canada anglais: éléments d'analyse», Nouvelles pratiques sociales, vol. 11, no. 1 (à paraître).

BROWNE, Paul Leduc (1999b). «The Neo-Liberal Uses of the Social Economy: Non-Profit Organizations and Workfare in Ontario", The Social Economy: Critique and Perspectives, sous la direction d'Eric Shragge et Jean-Marc Fontan, Montréal, Black Rose Books (à paraître).

BROWNE, Paul Leduc (1996). Love in a Cold World? The Voluntary Sector in an Age of Cuts, Ottawa, Centre canadien de politiques alternatives.

CAILLÉ, Alain (1995). «Pour ne pas rentrer à reculons dans le XXIe siècle. Temps choisi et don de citoyenneté", Contre l'exclusion. Repenser l'économie, s. la dir. de Juan-Luis Klein et Benoît Lévesque, Sainte-Foy, Presses de l'Université du Québec, 81-98.

CAMPBELL, Bruce et Maude BARLOW (1995). Straight Through the Heart: How the Liberals Abandoned the Just Society, Toronto, Harper Collins.

CASTEL, Robert (1995). Les métamorphoses de la question sociale. Une chronique du salariat, Paris, Fayard.

CHANDLER, William (1994). «La valeur du travail ménager au Canada, 1992», L'observateur économique canadien, Ottawa, Statistique Canada, avril.

CLARKE,Tony (1997). Silent Coup: Confronting the Big Business Takeover of Canada, Ottawa/Toronto, Centre canadien de politiques alternatives/Lorimer.

COMITÉ D'ORIENTATION (1996). Entre l'espoir et le doute, Rapport du Comité d'orientation et de concertation sur l'économie sociale, Québec, ministère de la Condition féminine, mai. 
CONSEIL NATIONAL DU BIEN-ÊTRE SOCIAL (1998). Profil de la pauvreté, 1996, Ottawa, Ministère des travaux publics et des services gouvernementaux.

DUSCHENE, Doreen (1989). Giving Freely: Volunteers in Canada, Ottawa, Statistique Canada, no. 71-535 au catalogue.

FAVREAU, Louis et Benoît Lévesque (1996). Développement économique communautaire. Économie sociale et intervention, Sainte-Foy, Presses de l'Université du Québec.

FAVREAU, Louis (1989). Mouvement populaire et intervention communautaire de 1960 à nos jours: Continuités et ruptures, Montréal, Centre de formation populaire/Éditions du fleuve.

FINLAYSON, Geoffrey (1994). Citizen, State and Social Welfare in Britain, 1830-1990, Oxford, Clarendon Press.

FREDERICK, Judith A. (1993). A Comparison of Results from the General Social Survey on Time Use1986 and 1992, Ottawa, Statistique Canada, décembre.

FREDERICK, Judith A. (1992). Measuring Unpaid Work:The Canadian Experience, Ottawa, Statistique Canada.

GLOBE AND MAIL (1995). «Unpaid Work Worth at Least \$234 Billion», The Globe and Mail, le 21 décembre.

GORZ, André (1997). Misères du présent. Richesse du possible, Paris, Éditions Galilée.

GORZ, André (1988). Métamorphoses du travail. Quête du sens, Paris, Éditions Galilée.

GORZ, André (1978). Écologie et politique, Paris, Éditions du Seuil.

HALL, Michael, Tamara KNIGHTON, Paul REED, Patrick BUSSIÈRE, Don MACRAE, Paddy

BOWEN, Caring Canadians, Involved Canadians: Highlights from the 1997 National Survey of Giving, Volunteering and Participating, Ottawa, Statistique Canada, août.

HALL, Stuart et Martin JACQUES (s. la dir. de) (1983). The Politics of Thatcherism, London, Lawrence and Wishart.

HARVEY, Andrew S., Katherine MARSHALL et Judith A. FREDERICK (1991). Where Does Time Go? Ottawa, Statistique Canada.

HARVEY, David (1988). The Condition of Postmodernity, London, Blackwell.

JACKSON, Andrew (1997). The Future of Jobs, Ottawa, Centre canadien de politiques alternatives.

JACKSON, Andrew (1995). The Liberals' Labour Strategy and Its Consequences for Workers, Ottawa, Centre canadien de politiques alternatives.

JENSON, Jane, Rianne Mahon et Manfred Bienefeld (1993). Production, Space, Identity : Political Economy Faces the 21st Century, Toronto, Canadian Scholars' Press.

KELSEY, Jane (1995). Economic Fundamentalism, London, Pluto Press.

KOSHAR, Rudi (1986). Social Life, Local Politics and Nazism: Marburg, 1880-1935, Chapel Hill, University of North Carolina Press.

LAROCHELLE, Gilbert et Joseph Tremblay (1994). «L'employabilité : éthique et sémantique d'un néologisme", Revue canadienne de politique sociale, no 34.

LAVILLE, Jean-Louis (1997). «Un nouveau contrat social», Possibles, vol. 21, no 2, printemps, 10614.

LAVILLE, Jean-Louis (ed.) (1994). L'Économie solidaire. Une perspective internationale, Paris, Desclée de Brouwer.

LAVILLE, Jean-Louis, avec la collaboration de Rainer Uhm, Bernard Eme, Silvia Gherardi, Richard MacFarlane, Alan Thomas (1993). Les services de proximité en Europe, Paris, Syros. 
LEGRAND, Julian et Will BARTLETT (1993). Quasi-Markets and Social Policy, Londres, Allen \& Unwin.

LEMANN, Nicholas (1996). «Kicking in Groups», The Atlantic Monthly, avril.

LÉVESQUE, Benoît (1995). «Repenser l'économie pour contrer l'exclusion sociale : de l'utopie à la nécessité», Contre l'exclusion. Repenser l'économie, s. la dir. de Juan-Luis Klein et Benoît Lévesque, Sainte-Foy, Presses de l'Université du Québec, p. 17-44.

LÉVESQUE, Benoît et William A. NINACS (1997). The Social Economy in Canada: The Québec Experience, Montréal, Institut de formation en développement économique communautaire.

LINHART, Robert (1978). L'Établi, Paris, Éditions de minuit.

LIPIETZ,Alain (1989). Choisir l'audace. Une alternative pour le vingt et unième siècle, Paris, La Découverte. McQUAIG, Linda (1993). The Wealthy Banker's Wife: The Assault on Equality in Canada, Toronto, Penguin Books.

MARCUSE, Herbert (1964). One-Dimensional Man, Boston, Beacon Books.

MARTIN, L'honorable Paul (1994). Un nouveau cadre de la politique économique, Ottawa, ministère des Finances, octobre.

MAURUTTO, Paula (1998). Governing Charities: Church and State in Toronto's Catholic Archdiocese, 1850-1950, thèse de doctorat, Université York, Toronto, mai.

MÉSZÁROS, István (1998). «The 35 Hour Week», allocution prononcée dans le cadre du Congrès des sociétés savantes, Université d'Ottawa, juin.

MÉSZÁROS, István (1995). Beyond Capital, London, Merlin Press.

MÉSZÁROS, István (1970). Marx's Theory of Alienation, London, Merlin Press.

MISHRA, Ramesh (1990). The Welfare State in Capitalist Society. Policies of Retrenchment and Maintenance in Europe, North America and Australia, Toronto, University of Toronto Press.

OCDE (1994). L'étude de l'OCDE sur l'emploi: faits, analyse, stratégies, Paris, OCDE.

POLANYI, Karl (1957). The Great Transformation, Boston, Beacon Press.

PROVOST, Monique (1989). «L'employabilité et la gestion de l'exclusion du travail», Nouvelles pratiques sociales, vol. 2 , no. 2 .

PUTNAM, Robert (1996a). "The Strange Disappearance of Civic America», The American Prospect, no. 24, hiver.

PUTNAM, Robert (1996b). «Robert Putnam Responds», The American Prospect, no. 25, mars-avril.

PUTNAM, Robert (1995). «Bowling Alone :America's Declining Social Capital», Journal of Democracy, vol. 6, no. 1, janvier, p. 65-78.

PUTNAM, Robert (1994). Making Democracy Work : Civic Traditions in Modern Italy, Princeton, Princeton University Press.

PUTNAM, Robert (1993). «The Prosperous Community : Social Capital and Public Life», The American Prospect, no. 13, printemps.

QUARTER, Jack (1992). Canada's Social Economy. Co-operatives, Non-profits, and Other Community Enterprises, Toronto, Lorimer.

RALPH,Diana, André RÉGIMBALD et Nérée ST-AMAND (s. la dir. de) (1997). Open for Business, Closed to People: Mike Harris's Ontario, Halifax, Fernwood Publishing.

RCPS (1998). Revue canadienne de politique sociale, numéro spécial sur l'économie plurielle/welfare pluralism, no. 41 , printemps. 
RÉGIMBALD, André (1995). «Le budget Martin: des politiques économiques pensées ailleurs?», Revue canadienne de politique sociale, no. 35, printemps.

REKART, Josephine (1993). Public Funds, Private Provision :The Role of the Voluntary Sector,Vancouver, UBC Press.

RIFKIN, Jeremy (1994). The End of Work. The Decline of the Global Labour Force and the Dawn of the Post-Market Era, New York, Tarcher/Putnam

ROSANVALlON, Pierre (1981). La Crise de l'État-providence, Paris, Éditions du Seuil.

ROUSTANG, Guy, Jean-Louis Laville, Bernard Eme, Daniel Mothé, Bernard Perret (1996). Vers un nouveau contrat social, Paris, Desclée de Brouwer.

SALAMON, Lester (1994). «The Rise of the Non-Profit Sector», Foreign Affairs, juillet/août.

SCHELlENBERG, Grant et Christopher Clark (1995). Temporary Employment in Canada: Profiles, Patterns and Policy Considerations, Ottawa, Conseil canadien de développement social.

SHERIDAN, Mike, Deborah Sunter et Brent Diverty (1996). «Évolution de la semaine de travail: tendances dans les heures de travail hebdomadaires au Canada, 1976-1995", La population active, Ottawa, Statistique Canada, juin.

SKOCPOL, Theda (1996). «Unravelling from Above», The American Prospect, no. 25, marsavril, 20-25.

STANFORD, Jim (1997a). «Disappearing Deficits and Incredible Interest Rates: Canada's Hollow EconomicVictories», Alternative Federal Budget Papers 1997, Ottawa, Centre canadien de politiques alternatives, 219-274.

STANFORD, Jim (1997b). «Growth, Interest and Debt: Canada's Fall from the Fiscal Knife-Edge», Alternative Federal Budget Papers 1997, Ottawa, Centre canadien de politiques alternatives, 274292.

STANFORD, Jim (1995). «Bending Over Backwards : Is Canada’s Labour Market Really Inflexible?», Canadian Business Economics, automne 1995, 70-87.

STATISTIQUE CANADA (1998). «L'Aperçu statistique», L'Observateur économique canadien, décembre.

STATISTIQUE CANADA (1997). Le point sur la population active. Les heures de travail, Ottawa, Statistique Canada, été.

URSEL, Jane (1992). Private Lives, Public Policy. 100 Years of State Intervention in the Family, Toronto, Women's Press.

VAILLANCOURT,Yves (1997). Vers un nouveau partage des responsabilités dans les services sociaux et de santé: rôles de l'État, du marché, de l'économie sociale et du secteur informel, avec la collaboration de Christian Jetté, Montréal, Université du Québec à Montréal, Cahiers du Larepps 97/05.

VOSKO,Leah (1999). «Workfare Temporaries:Workfare and the Rise of the Temporary Employment Relationship in Ontario», Revue canadienne de politique sociale, no. 42, automne 1998.

WARING, Marilyn (1996). Three Masquerades. Essays on Equality, Work and Human Rights, Auckland, Auckland University Press with Bridget Williams Books.

WARING, Marilyn (1990). IfWomen Counted. A New Feminist Economics, New York, Harper Collins. 


\section{Note}

1. En partant de l'Enquête sur la population active de Statistique Canada, on peut établir le taux de chômage moyen de 1946 à 1955 à 3,16\%; de 1956 à 1965 à 5,51\%; de 1966 à 1975 à $5,91 \%$, de 1976 à 1985 à 9,1\%; et de 1986 à 1995 à 9,47\% (Akyeampong 1996:10). 